\title{
SsRNA-based Immunomodulator CV8102
}

National Cancer Institute

\section{Source}

National Cancer Institute. sSRNA-based Immunomodulator CV8102. NCI Thesaurus.

Code C150401.

A 547 nucleotide (nt), noncoding, uncapped sing le-stranded RNA (ssRNA) containing several polyU-repeats complexed with a polymeric carrier formed by disulfide-crosslinked cationic peptides, with potential immunostimulating activity. Upon intratumoral injection, the ssRNA in CV8102 activates toll-like receptors (TLRs) and retinoic acid-inducible gene I (RIG I; RIG-I; DDX58). This stimulates a T-helper type 1 cell (Th1) response, the production of a variety of pro-inflammatory cytokines and chemokines, and activates a systemic cytotoxic-T-lymphocyte (CTL)-mediated immune response against the tumor cells when simultaneously exposed to tumor-associated antigens (TAAs). The cationic carrier peptides protect the ssRNA from RNase degradation. 